\title{
Analysis of the Transient Process in Underwater Spark Discharges
}

\author{
I. V. Timoshkin, R. A. Fouracre, M. J. Given, and S. J. MacGregor \\ HVT Research Group, Institute for Energy and Environment, Department of Electronic and Electrical Engineering \\ University of Strathclyde, Royal College Building, 204 George St, Glasgow, G1 1XW, UK
}

\begin{abstract}
If water is stressed with a voltage pulse having a rise time of tens of nanoseconds which creates a sufficiently high electric field, streamers develop and a highly conductive channel forms between the electrodes. The intense Joule heating of the plasma in the channel results in the collapse of its electrical resistance from a few Ohms to a few tens of milliOhms with the behavior of the collapse depending on the parameters of the discharge circuit. The rapid decrease of the resistance occurs during the first quarter of the current oscillation in the circuit. During this time, the pressure inside the channel rises to several GPa, causing the channel to expand in water with a velocity of 100 to $1000 \mathrm{~m} / \mathrm{s}$ driving a high power ultrasound pulse. In the present paper, a phenomenological model is discussed which describes the dynamics of the resistance of underwater spark discharges during its initial stage and allows the pressure in the acoustic pulse to be obtained. The model is based on the plasma channel energy balance equation used by Braginskii and links the hydrodynamic characteristics of the channel and the parameters of the electric driving circuit. The dynamics of the transient cavity during the dissipation of the electrical energy in the plasma channel is described and the analytical results are compared with experimental measurements of the current in the electrical circuit and the acoustic pulse profiles radiated by the transient cavities.
\end{abstract}

\section{INTRODUCTION}

Streamer breakdown of water and the dynamics of the fast transient cavity developed as a result of this process has been the subject of research by several investigators. The interest in this subject is driven particularly by the use of water as a medium in high voltage switching devises employed in pulsed power systems [1], and the generation of wide band high power ultrasound (HPU) pulses through underwater spark discharges.

The resistance of the plasma cavity, $R_{p l}(t)$, decreases significantly from a relatively large value at the moment when the first streamer bridges the inter-electrode gap forming a narrow conductive channel which results in the appearance of a high conduction current, $I(t)$, to a much lower plateau value at maximum current. Potentially, this transient resistance could by obtained from current and voltage waveforms, but the rapid collapse of the voltage together with the problems of separating the resistive and inductive components of the spark channel impedance makes measurements of the time-dependent resistance extremely difficult $[2,3]$. Information on the transient resistance of the plasma channel is important in the design of water-dielectric switches to allow the evaluation and reduction of energy dissipation, or in water spark discharges used in practical applications for the optimization of energy release. To calculate the parameters of the expanding plasma channel and its transient resistance together with the HPU pulse profile, an analytical model has been proposed. It can be shown that the results of calculations using this model are in good agreement with experimental measurements.

\section{ANALYTICAL MODEL}

To describe the time-dependent resistance of the plasma channel in water during its decreases from its initial values of a few Ohms to its plateau resistance of a few tens of milliohms an analytical model based on the Braginskii hydro-dynamic energy balance equation and an empirical link between the plasma channel resistance and its internal energy is used. The Braginskii equation, discussed in [4], states that the electrical energy released in the plasma channel through the Joule heating is divided between the internal energy of the plasmavapor mixture inside the transient cavity, $W_{\text {in }}(t)$, and the mechanical work done by the expanding cavity against water:

$$
\frac{d W_{i n}(t)}{d t}+P \frac{d V(t)}{d t}=R_{p l}(t) I(t)^{2}
$$

where $V(t)$ is the volume of the plasma channel and $P$ is the pressure in the channel. Energy losses due to light emission and heat conduction have been neglected in Equation (1).

Assuming that the electrical behavior of the system can be approximated by a series $L C R$ circuit. A Kirchhoff equation can be written in terms of the current:

$$
\frac{d I(t)^{2}}{d t^{2}}+\frac{1}{L} \frac{d}{d t}\left(\left(R_{p l}(t)+R_{s t}\right) I(t)\right)+\frac{I(t)}{L C}=0
$$

where $C$ and $L$ are the capacitance and the inductance of the pulsing circuit, and $R_{s t}$ is the constant stray resistance.

To link the hydro-dynamic energy balance equation (1) with the Kirchhoff equation (2), the empirical expression for the plasma channel resistance used proposed in [5] has been used:

$$
R_{p l}(t)=\frac{A_{s p} \ell^{2}}{W_{i n}(t)}
$$

where $A_{s p}$ is a spark constant which characterises the content of the spark breakdown channel, and $\ell$ is the channel length. Expression (3) has been validated experimentally and it was 
found that $A_{s p}$ changes insignificantly during the first half cycle of the current oscillation and has a value $A_{s \mathrm{p}} \approx 2.5 \cdot 10^{4} \mathrm{~V}^{2} \mathrm{~s} / \mathrm{m}^{2}$ for a discharge with the energy of $\sim 2.6 \mathrm{~kJ}$ [6]. This value of the spark constant has been used in the present model.

The parameter $W_{\text {in }}(t)$ can be derived using:

$W_{\text {in }}(t)=\frac{P(t) V(t)}{\gamma-1}$

It has been assumed that the ionized water components and vapor in the spark discharge channel behave adiabatically as an ideal gas with a constant ratio of specific heats $\gamma=1.3[7,8]$.

Equations (1)-(4) allow the time-varying resistance and radius of the spark channel to be derived together with the current oscillating in the pulse driving circuit. To describe the radiated acoustic pulse as a linear acoustic approximation, the equation for the pressure, $P_{w}(t)$, in a compression wave propagating in water [7] can be used:

$P_{w}(\bar{t})=P_{0}+\frac{\rho_{0}}{4 \pi r} \frac{d^{2} V(\bar{t})}{d t^{2}}-\frac{\rho_{0}}{2\left(4 \pi r^{2}\right)^{2}}\left(\frac{d V(\bar{t})}{d t}\right)^{2}, \bar{t}=t-\frac{r}{c_{0}}$

where $P_{0}$ is the external hydrostatic pressure, $c_{0}$ is the speed of sound in water, and $r$ is the distance between the spark discharge channel and an observation point in water.

To allow numerical solutions of equations (1)-(5), it is convenient to transform them into a dimensionless form. This transformation can be done by the introduction of the following normalization parameters:

$$
\begin{aligned}
& A=\frac{r_{c h} I_{0}^{2} \tau_{0}}{E_{0}}, B=\frac{\rho_{0}}{P_{0}} \frac{a_{0}^{2}}{\tau_{0}^{2}}, r_{c h}=\frac{N}{P_{0} a_{0}^{2}}, \\
& N=\frac{A_{s p}(\gamma-1) \ell}{\pi}, M=\frac{\tau_{0}^{2}}{\rho_{0} a_{0}^{2}}, a_{0}=\left(\frac{3(\gamma-1)}{4 \pi} \frac{\tau_{0}^{2} E_{0}}{\rho_{0}}\right)^{\frac{1}{5}} \\
& I_{0}=U_{0} \sqrt{\frac{C}{L}}, \tau_{0}=\sqrt{L C}, R_{c r i t}=2 \sqrt{\frac{L}{C}}, E_{0}=\frac{1}{2} C U_{0}^{2}
\end{aligned}
$$

where $U_{0}$ is the charging voltage. $a_{0}$ is the characteristic discharge length introduced in [9] which gives an approximate value of the radius of the spherical transient cavity at the end of the energy deposition stage of the spark discharge if $E_{0}$ is the total energy delivered to the spark channel.

Using the normalization parameters (6), the energy balance equation (1), the Kirchhoff equation (2) and the equation for the pressure pulse in water (5) can be re-written as:

$$
\begin{aligned}
& \frac{d^{2} z(x)}{d x^{2}}+2 \frac{r_{c h}}{R_{c r i t}} \frac{1}{p(x) y(x)}\left\{\frac{d z(x)}{d x}-z(x)\left(\frac{1}{p(x)} \frac{d p(x)}{d x}+\right.\right. \\
& \left.\left.\frac{2}{y} \frac{d y(x)}{d x}\right)\right\}+2 \frac{R_{s t}}{R_{c r i t}} \frac{d z(x)}{d x}+z(x)=0
\end{aligned}
$$

$$
\frac{d^{2} y(x)}{d x^{2}}=\frac{1}{y^{2}}\left\{\frac{f(x)}{y(x)^{2}}-\frac{3}{2} \gamma y(x)\left(\frac{d y(x)}{d x}\right)^{2}-M P_{o} y(x)\right\}
$$

where $f(x)=A \int \frac{z(x)^{2}}{p(x) y(x)^{2}} d x$, and

$$
\begin{aligned}
& p_{w}(\bar{x})=1+\frac{\rho_{0}}{P_{0}}\left(\frac{a_{0}}{\tau_{0}}\right)^{2}\left(\frac{a_{0}}{r_{*}}\right) y(\bar{x})\left(y(\bar{x}) \frac{d^{2} y(\bar{x})}{d \bar{x}^{2}}+2\left(\frac{d y(\bar{x})}{d \bar{x}}\right)^{2}\right) \\
& -\frac{1}{2} \frac{\rho_{0}}{P_{0}} \frac{a_{0}^{6}}{\tau_{o}^{2} r_{*}^{4}}\left(\frac{d y(\bar{x})}{d \bar{x}}\right)^{2} y(\bar{x})^{4}
\end{aligned}
$$

Here, $x$ is dimensionless time, $z(x)$ is dimensionless current, $y(x)$ is the dimensionless radius of the plasma channel, $p(x)$ is the dimensionless pressure in the channel, $p_{w}(\bar{x})$ is the dimensionless pressure in the acoustic pulse at the dimensionless distance, $r_{*}$, and the function $f(x)$ determines the electrical energy dissipated in the plasma due to the Joule heating. The initial conditions for the solution can be set as follows: in the electrical circuit at the initial time instant, $x=0$, the current is absent and the total charge is stored in the capacitance of the electrical circuit:

$z(0)=0,\left.\quad \frac{d z(x)}{d x}\right|_{x=0}=1$

For the plasma channel at the time instant $x=0$ the plasma channel has a radius $y_{0}$ and its expansion velocity is zero:

$y(0)=y_{0},\left.\quad \frac{d y(x)}{d x}\right|_{x=0}=0$

The model is not sensitive to the choice of the initial radius of the streamer as it evolves quickly during the energy deposition stage and its behavior is determined by the parameters of the energy contribution into the plasma channel.

\section{EXPERIMENTAL SET-UP}

To compare the analytical model with experimental results, measurements of the current waveforms for spark discharges in water and the HPU pulse profiles produced by these discharges were conducted. The spark discharges were generated in tap water by a pulsed power supply designed to deliver HV pulses with magnitudes up to $35 \mathrm{kV}$ and energies up to $1 \mathrm{~kJ} /$ pulse. The spark discharge source has the inter-electrode gap of $5 \mathrm{~mm}$. The acoustic pulses were detected with a ValpeyFisher VP-1093 Pinducer and the discharge waveforms were obtaining using a high voltage probe and a resistive current shunt. A detailed description of the experimental set-up including current and voltage traces and HPU pulses profiles can be found in [10]. 


\section{Results}

\section{A. Model Verification}

The model presented in the Section II has been verified using the phenomenological approach proposed in [9]. If the behavior of dissipation of the electrical energy in the plasma channel is known, Equation (1) could be solved directly, without involvement of the Kirchhoff equation (2). The energy dissipation function could be obtained from the experiments using the discharge waveforms. As was shown in [11], the breakdown current waveforms for the underwater spark discharges generated by the pulse driving circuit and the electrode system match the analytical curves for under-damped current oscillations in a series $L C R$ circuit with a constant resistance. The value of the constant resistance, $R_{\text {const, }}$ associated with the plasma channel could be calculated by subtracting the value of the stray circuit resistance, $R_{\text {stray }}$, derived under short circuit conditions, from the values of resistance derived from the spark discharge waveforms.

After integration and simplification introduced in [9] Equation (1) can be re-written as:

$$
\frac{d^{2} V(t)}{d t^{2}} V(t)=4 \pi \frac{\gamma-1}{\rho_{0}} a(t) E(t)
$$

where $a(t)$ is the channel radius and $E(t)$ is the electrical energy dissipated in the plasma channel. For the current oscillations in a series $L C R$ circuit with constant resistance $R_{\text {const, }} E(t)$ takes the form:

$$
E(t)=\int R_{\text {const }} I_{1}^{2} \exp (-2 \alpha t) \sin ^{2}(\omega t) d t
$$

where parameters $I_{1}, \alpha, \omega$ can be obtained from the experimental current waveforms.

For spark discharges with electrical energies between $151.7 \mathrm{~J}$ and $916.7 \mathrm{~J}$, the constant resistance values, $R_{\text {const, }}$ are in the range of $103 \mathrm{mOhm}$ for the lowest pulse energy to $37 \mathrm{mOhm}$ for the highest pulse energy [11]. These values of $R_{\text {const }}$ together with the values of stray resistance are listed in Table I.

TABLE I. RESISTANCES $R_{\text {CONST }}$ AND $R_{S T R A Y}$

\begin{tabular}{|c|c|c|}
\hline Energy, $\mathrm{J}$ & $R_{\text {const, }}$ mOhms & $R_{\text {stray, }}$ mOhms \\
\hline 151.7 & 103 & 555.5 \\
\hline 281.5 & 77.5 & 407.2 \\
\hline 451.5 & 64.9 & 360.7 \\
\hline 605.2 & 49 & 325.1 \\
\hline 747.0 & 46.9 & 308.0 \\
\hline 916.7 & 37.1 & 302.4 \\
\hline
\end{tabular}

Equations (12) and (13) have been normalized using appropriate parameters from Expressions (6) and solved numerically for the constant resistances listed in Table I and corresponding experimental parameters $I_{l}, \alpha, \omega$. The expected amplitudes of the acoustic pressure pulses $500 \mathrm{~mm}$ away from the discharges have been calculated using Equation (9).
An alternative approach was also used where Equations (7)(11) have been solved using the same values of breakdown voltage, $U_{0}$, capacitance, $C$, inductance, $L$, and stray resistance, $R_{\text {stray, }}$, as for the discharges listed in Table I but assuming that the resistance of the plasma channel varies with time. Again, the amplitudes of the pressure pulses $500 \mathrm{~mm}$ away from the spark electrodes have been obtained.

The comparison between the phenomenological approach with the defined energy dissipation function (Equation (13)) and the analytical model with a time-dependant plasma channel resistance is shown in Figure 1.

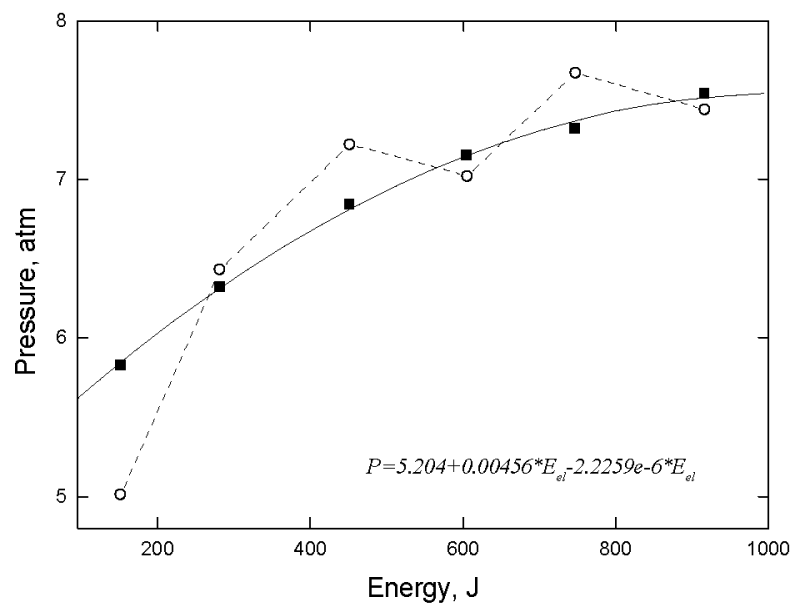

Fig. 1. Pressure pulse amplitudes calculated by Equations (12)-(13) (open points connected with dash line) and by Equations (7)-(11) (solid squares) Solid black line shows polynomial fitting of squares.

It can be seen that the model with the dynamic resistance gives values of acoustic amplitudes similar to those calculated using the model with the experimental energy dissipation function. The graph also shows that the calculated pressure in the radiated pulse increased only by $\sim 23 \%$ while the electrical energy available to the discharge increased by $\sim 83 \%$. Measurements of acoustic pulse amplitudes as a function of energy available confirms that the pressure in the HPU pulses does not increase linearly with increasing electrical pulse energy [10].

\section{B. Calculations Using the Model}

Equations (7)-(11) have been used to calculate the expected behavior of the plasma channel resistances and current waveforms. An example of such calculations is shown in Figure 2 for a discharge with an energy of $747 \mathrm{~J}$.

As can be seen, the resistance of the channel reduces significantly from a few Ohms to $\sim 31 \mathrm{mOhm}$ during the first quarter of the current oscillation. The current waveform, $I(t)$, obtained from the model (solid line) matches well the waveform, $I(t)_{\text {exp }}$, plotted using the experimental values of the parameters $I_{1}, \alpha, \omega$ averaged over five discharges and the standard expression for the under-dumped current in a series $L C R$ circuit (dash-dotted line). The difference in the maximum current values between these two waveforms is less than $12 \%$. 


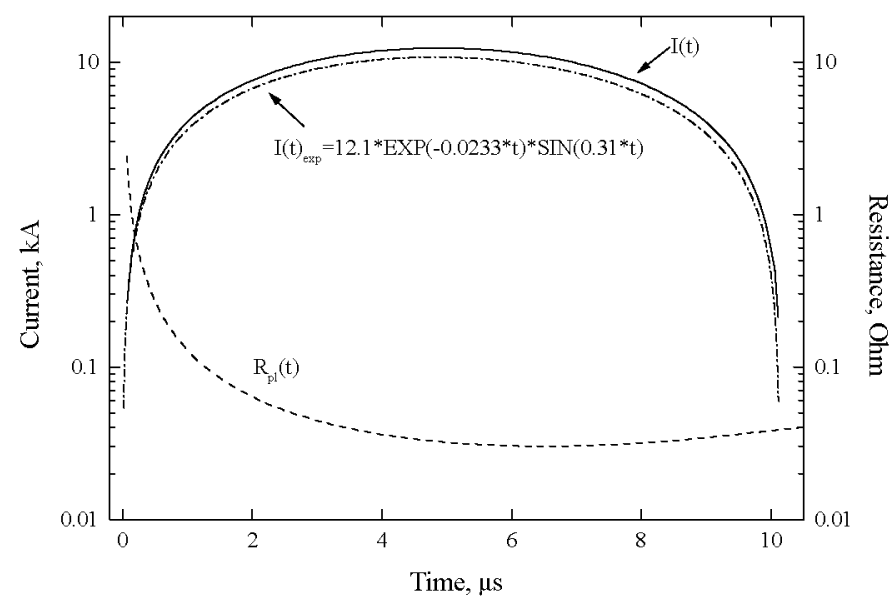

Fig. 2. Current waveforms obtained using the analytical model for $747 \mathrm{~J}$ discharge, $I(t)$, solid line; and from experimental measurements, $I(t)_{\text {exp }}$, dash-

dotted line. $R_{p l}(t)$ is time-depended resistance of the plasma channel.

The analytical model has also been used to calculate the expected maximum pressure amplitude in the HPU pulses produced by the different sets of capacitors and charging voltages used in the study presented in [10] and [12]. The calculated peak pressures have been compared with the experimental results reported in these papers and the result of this comparison is shown in Figure 3.

The pressure values calculated by the analytical model are shown in this graph by solid points connected with solid lines, while the experimental pressure values are shown by open points. Each set of points (open and solid) corresponds to the same value of the capacitance in the pulsed power system. Changes in the electrical pulse energy were achieved by varying the charging voltage. The analytical results shown in Figure 3 match the experimental peak pressures with reasonable accuracy and show that increasing the pulse energy does not result in a linear increase in the acoustic pressure. In addition, the results in Figure 3 demonstrate that the acoustic energy of HPU pulses generated with common energies is dependent upon the voltage pulse magnitude for constant energy per pulse.

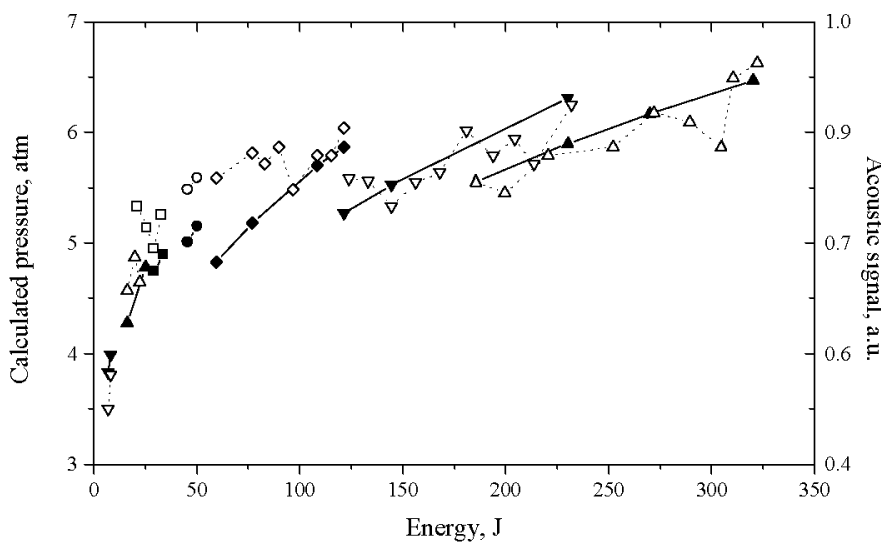

Fig. 3. Calculated peak pressure and experimental acoustic signals registered by Pinducers [10], [12].

\section{Conclusions}

The analytical approach presented in this paper allows the functional behavior of the plasma channel resistance to be obtained for underwater spark discharges. Although the model is based on the phenomenological description of the plasma channel dynamics and its resistance and requires the knowledge of the spark constant, the results obtained using this approach provide a reasonable agreement with experimental measurements.

It was shown that pressure in the HPU pulses generated by underwater spark discharges with the constant inter-electrode gap spacing does not increase significantly with increase in the pulse electrical energy. However, increasing the charging voltages produces greater amplitudes of the acoustic signals at the same pulse energy. This approach is therefore preferable for practical HPU applications. The model could be used in the optimization of the parameters of the pulsed power circuits for various applications of underwater spark discharges such as the comminution and recovery of waste materials [13].

\section{REFERENCES}

[1] J. R. Woodworth, J. M. Lehr, J. Elizondo-Decanini et al., "Optical and pressure diagnostics of 4-MV water switches in the Z-20 test facility", IEEE Trans. on Plasma Science, v. 32, no. 5, 2004, pp.1778-1789.

[2] J. P. VanDevender "The resistive phase of a high-voltage water spark", $J$. Appl. Phys. v.49, no.5, 1978, pp.2616-2620.

[3] J. M. Lehr, J. P. Corley, J. E. Elizondo et al., "Multi-megavolt switching in water: considerations for the Z-R machine", Proc. Int. Conf. on Elect. Insul. and Diel. Phenom., 2002, pp.554-557.

[4] T. G. Engel, A. L. Donaldson, M. Kristiansen, "The pulsed discharge arc resistance and its functional behavior", IEEE Trans. on Plasma Science, v.17, 1989 , pp.323-329.

[5] V. Ts. Gurovich, A. Grinenko, Ya.E. Krasik, J. Felsteiner, "Simplified model of underwater electrical discharge", Phys. Rev. E, v.69, 2004, pp.036402-1-6.

[6] E. V. Krivitski, V. V. Shamko, "Similarity of underwater spark discharges", Sov.Phys.-Tech.Phys., v.17, n.1, 1972, pp.62-65.

[7] E. Gidalevich, R. L. Boxman, S. Goldsmith, "Hydradynamic effects in liquids subjected to pulsed low current arc discharges", J. Phys.D: Appl. Phys., v.37, 2004, pp.1509-1514.

[8] G. A. Shneerson, "Estimation of the pressure in a "slow" spark discharge in a cylindrical water-filled cavity", Technical Physics, v.48, n.3, 2003, pp.374-375.

[9] K. A. Naugol'nykh and N. A. Roi, Spark Discharges in Water, Nauka, Moscow, 1971 (translation, Foreign Technology Division, Wright-Patterson $\mathrm{AFB}, \mathrm{OH}, 1974)$

[10] J. W. Mackersie, I. V. Timoshkin, S. J. MacGregor, "Generation of high power ultrasound by spark discharges in water", IEEE Trans. on Plasma Science, v.32, no.5, 2005, p.2055-2061.

[11] I. V. Timoshkin, S. J. MacGregor, M. J. Given, R. A. Fouracre, "Gasfilled transient cavities generated by spark discharges in liquids", Proc. $16^{\text {th }}$ Int. Conf. on Gas Discharges and Their Applications, China, Sept. 2006, in press.

[12] S. J. MacGregor, J. W. Mackersie, "The development of high power ultrasound for acoustic source and industrial applications", EPSRC project GR/R41095/01, Final Report, 2004

[13] M. P. Wilson, L. Balmer, M. J. Given, S. J. MacGregor, I. V. Timoshkin, "Optimisation of the spark gap parameters for high power ultrasound applications", Proc. $27^{\text {th }}$ Int. Power Modulator Conference, Washington DC, May 2006, in press. 